\title{
Adaptive Product Review Classification Engine (APRCE) for Social Network Product Review Evaluation
}

\author{
Anjani Chanji \\ Punjabi University \\ Regional Centre for \\ Information Technology \\ and Management, \\ Mohali, Punjab, India
}

\author{
Rekha Bhatia \\ Punjabi University \\ RegionalCentre for \\ Information Technology \\ and Management, \\ Mohali, Punjab, India
}

\begin{abstract}
The product review classification is the process of automatic categorization of the review data based upon the specific products. The product review classification helps the techniques for analysis of the product reviews and to classify them according to the polarity evaluation. The product review classification has been utilized for the comparative analysis of the two similar products to facilitate the customers to make their decision on the basis of the public opinion and choice. In this paper, the product review classification model has been implemented with the supervised modeling, which evaluates the multiple keyword lists for the evaluation of the polarity across the input review data for the different products of the similar categories. The proposed model performance has been evaluated in thevarious domains such as text processing errors, compression types, recall, precision, polarization accuracy, etc. The proposed model has been found efficient in the terms of all of the performance parameters in comparison with the existing models.
\end{abstract}

\section{Keywords}

Product reviews classification, Polarization, Sentence

Compression, Review Classification.

\section{INTRODUCTION}

The vigorous growth of internet and social media; peoples life have changed with more and more people sharing their views online whether it be social networking sites, blogs, shopping sites, forums etc. It enables user to present views about the product and read about other people's opinion about the product. Online shopping portals like amazon, snapdeal, ebay, flipkart etc. people in addition to buy the product can share their opinions about the product. That would hence benefit other people to make up their mind about whether to purchase the product or not. Large amount of data is collected over such platforms over the internet in the database of different platforms also known as Big Data. Business companies employ huge number of technocrats to analyze user views about their produced goods which is further helpful to get reviews of their product and improvising their products. So keeping a watch on the platforms is vital as to know the real scenario among the people; how they are responding to the event or brand. This big data analysis eases the user to predict the sentiments of user over the particular course of event.

Sentiment analysis refers to utilize natural language processing, analysis of text and computational linguistic to determine and extract subjective information in the source text. Sentiment is determining the perception, reaction or view point. Sentiment analysis is the field of opinion mining which implies sentiments towards certain entities and attributes. Sentiment analysis is one of the most trending research areas now days. With the rapid growth of internet the online review sites; the main characteristic of posted articles describes the sentiments of the people about the characteristics product that can be positive negative or neutral. Thus sometimes sentiment analysis is also known as polarity classification. These sentiment classifications are very beneficial in business intelligence.

Sentiment analysis can be performed using various approaches like lexicon based approach, machine learning methods, supervised, semi-supervised, unsupervised approaches. Lexical based approach is either dictionary based or corpus based. In dictionary based the sentiment words have given some polarity scores. In corpus based probability of word co-occurring with negative or positive words form wide range of database. Some examples of supervised approach algorithms are naïve Bayes, Support Vector Machine (SVM), K-Nearest Neighbor, Neural network that is the approach that require labeled training set and test data. Unsupervised approach is emotion based and doesn't require training data.

This paper proposes a supervised approach algorithm APRCE an Adaptive Product Review Classification Engine for Social Network Product Review Evaluation. The proposed model based upon the supervised polarity evaluation and supervised categorization of the message data obtained from the social network for the input product.The algorithm calculates the sentiment in the social threads with positive or negative messages. The sentiment analysis in the proposed model focuses upon the classification and categorization for the feature classification along with overall automatic summarization.Then the proposed model performance has been evaluated in the various domains such as text processing errors, compression types, recall, precision, polarization accuracy, etc.

In this section of introduction (section 1) the overview of the sentiment analysis model and method has been discussed. The rest of this paper is organized as follows: In section literature review (section 2) a brief review about various related work on sentiment analysis is studied and presented. In Experimental design (section 3) describes the experiment analysis of proposed model. In Result analysis section (section 4) describes the result and its analysis. Finally the section 5 concludes the paper.

\section{LITERATURE REVIEW}

W Che et al.have proposed a sentence compression model for that is for aspect based sentiment analysis. In this model sentence is compressed before the sentiment analysis step. Sentence compression 'SENTCOMP' is an model that discards the unnecessary words before sentiment analysis process and thus compacts the sentence that is uncomplicated 
to parse and have an outrageous performance for sentiment analysis. The accuracy achieved by using this model is $90 \%$. As 'Sent comp' model discards the repeated duplicate words, unwanted words but it sustain polarity based information that is necessary for sentiment analysis.

A Esuli et al.have proposed an SentiWordNetthat that is an lexical resource for mining opinions .WordNetsynset have scores the following 'obj(s)', 'Neg(s)','Pos(s)' that have some numerical values that presents how negative, positive, objective is hold in synset. It comes with an commendable GUI. Eight Ternary classifiers are trained so that three scores for synset are obtained. Training set and learning device for respective ternary classifier is specific and hence specific classification outcome of the related synset is produced. Scores for opinions are calculated by normalization on the basis of assigned labels. Synset will have maximal score when every ternary classifier assigns same label to synset otherwise the score will be proportional to ternary classifiers.

Minqing Hu et al. have proposed a model that summarizes opinion based on their features. In the proposed work,they have worked on the features that the customers have reviewed. Firstly the proposed work identifies what are the features of the product that the consumer have reviewed on are identified and thentheir scores are assigned on the basis of the times of frequency in the review. For every feature had been reviewed; the positive and negative opinions are determined. Than lastly opinion summarization is implemented by summarizing the opinions for extracted characteristics in two classes that is positive and negative.

B. Pang et al. had worked on the movie reviews for sentiment analysis. They worked upon three machine learning methods Naïve Bayes, Maximum Entropy Classification and Support vector Machine. They have focused on the Sentiment classification problem rather than the topic based classification. The Support vector Machine had outperformed the three algorithms but the dissimilarity is not much when compared with the human generated baselines. Even when compared with topic based classification the accuracy is not as much. Here authors have faced the problem of feature identification.

S Mukherjee et al. has proposed sentiment analysis for feature based product reviews. Their main goal is to determine the possible feature by utilizing their correlation. The model get backs opinion expression that illustrates the features specified by the user. The model has showed better accuracy and had outturned in performance in all the elected baselines. Under supervised classification model performs much better with huge margin. The main shortcoming of the system is that it doesn't train data that is domain specific and hence can't analyze the domain dependent sentiments.

X Fang et al.have worked on sentiment analysis of product reviews on amazon data set. Basically their work focuses on polarity categorization of sentiments. For this polarity categorization of sentiments they have presented feature vector categorization method. The method is implemented at both sentence and review level. They have used three different classifiers that are naïve based, random forest and support vector machine. The review level categorization needs to be improved as it doesn't perform well for implicit sentence; the sentence with neutral words. So here review level categorization needs to be improved by extracting more features and grouping them into feature vector.
The section 3 describes the experimental design of the Adaptive Product Review Classification Engine (APRCE) for Social Network Product Review Evaluation

\section{EXPERIMENTAL DESIGN}

Sentiment Analysis is the technique to analyze the emotion from a text document, message, or similar content. Sentiment Analysis algorithms are used to predict the public opinion on various issues getting discussed in the social network threads. Hence, it is also called opinion mining or emotion mining. In this paper, we have proposed the algorithm to calculate the sentiment in the social threads with positive or negative messages. At first, the messages are scanned for their positivity or negativity. Then, the messages are automatically classified according to their product feature and re-analyzed for further emotion. The algorithm uses the weighted dictionary matching to analyze the sentiment in the messages. The social thread is also recording the results on the basis of every user taking part in the social discussion thread. The results have shown the high accuracy of $95.50 \%$. The accuracy has been measured on the basis of type 1 and type 2 statistical errors. The sentiment analysis in the proposed model focuses upon the classification and categorization for the feature classification along with overall automatic summarization. The involvement of the specialized keyword based opinion mining with the selective feature selection for the improvement of the product classification and intensity calculation. The proposed rule-based feature relation and classification model deeply analyzes the text features for the automatic classification of the product reviews obtained from the online sources. The proposed model review classification model algorithm is detailed in the following section

Algorithm 1: Proposed Feature Extraction and Review Classification Model

1. Load the social network review data for the specific product $\rightarrow \mathbf{T r}$

2. Run the iteration for each message in the database Tr

3. Perform the N-gram analysis over the message $\mathbf{I}$ using the supervised tokenization based upon dictionary matching

4. Load the STOPWORD list to remove the non-polar words from the message I

5. Run the supervised classification for product feature classification (PFC) using the specialized knowledge data

6. Calculate the message polarity using the PFC model

7. Acquisition of the sentiment and expression classification (SEC) knowledge data

8. Calculate the message score after comparing it with SEC data

9. Categorize the input message I using the polarity score

10. Increment the resulting indices for density evaluation of product feature indexing

a. If polarity score is higher than zero

i. Increment the value of positive index

b. If score is equal to zero

i. Increment the value of neutral index

c. If score is lower than zero

i. Increment the value of negative index 
11. Load product review summarization (PRS) knowledge data

12. Prepare the summarization content according to the sentiment report

The sentiment analytical model involves the various core steps to evaluate the polarity of the input message I, which is evaluated using the positive, negative and neutral messages. The proposed model of sentiment analysis uses the supervised learning method for the evaluation of the sentiment analytical model. The proposed model algorithm is defined as following:

Algorithm 2: Detailed Explanation of Sentiment Analysis Model

13. Obtain the data from the social network thread $\mathbf{T r}$

14. Extract the list of users $\mathbf{U}$ from the social networking thread

15. Extract $\mathbf{N}$ number of Message $\mathbf{M}$ using dictionary based tokenization

16. Load the STOPWORD list which contains the nonpolar words

17. Load the polarity lists containing the polarity wordlists

18. Calculate word weight score to measure the sentiment Sn

19. Count the final sentiment score $\mathbf{S}$ of each message (Positive/Negative)

20. Calculate sentiment score for tokenized message number $\mathbf{N}$

21. Calculate the polarity score by applying the similarity evaluation against the polarity lists

22. If polarity score higher than 0

a. Assign the tag 'positive' to the message

b. Increment the number of positive messages by one

23. If polarity score lower than or equal to 0

a. Assign the tag 'negative' to the message

b. Increment the number of negative messages by one

The section 4 describes the result analysis obtained by implementing the proposed model and compared it with the existing model.

\section{RESULT ANALYSIS}

The proposed model has been designed to analyze the public opinion the mobile phones. The public reviews on the handsets from the various manufacturers have been obtained from online sources such as facebook, twitter and gsmarena. The public reviews has been kept in the excel format and the loaded in the runtime memory using the MATLAB module for Microsoft document handling. The user review data has been automatically classified for the product features and then the emotion analysis has been performed on the classified messages and the calculation emotion is added to the corresponding category under the product review classification. The product review classification has been done using the dictionary defined for the various product features in the mobile phones. The result snapshots have been shown in the following section:
Table 4.1: A table of Precision and Recall for various densities of messages

\begin{tabular}{|c|c|c|c|}
\hline Sr. No. & $\begin{array}{c}\text { Total } \\
\text { Number of } \\
\text { Messages }\end{array}$ & $\begin{array}{c}\text { Recall } \\
(\mathbf{T P} / \mathbf{T P}+\mathbf{F N})\end{array}$ & $\begin{array}{c}\text { Precision } \\
\mathbf{T P} / \mathbf{T P}+\mathbf{F P}\end{array}$ \\
\hline 1 & 10 & $100 \%$ & $100 \%$ \\
\hline 2 & 100 & $100 \%$ & $94.90 \%$ \\
\hline 3 & 500 & $100 \%$ & $97.57 \%$ \\
\hline 4 & 1000 & $100 \%$ & $98.58 \%$ \\
\hline 5 & 2000 & $99.80 \%$ & $98.52 \%$ \\
\hline 6 & 5000 & $99.91 \%$ & $98.86 \%$ \\
\hline 7 & 10000 & $99.18 \%$ & $98.85 \%$ \\
\hline AVERAGE & - & $99.84 \%$ & $98.18 \%$ \\
\hline
\end{tabular}

The proposed model has been evaluated for the various performance parameters (table 4.1), which show its effectiveness using the Recall and Precision values. The Precision and Recall of the proposed model is quite higher making the proposed model highly efficient in the terms of sentiment analysis, product feature classification and automatic summarization.

Table 4.2: The table of comparison between existing and proposed model

\begin{tabular}{|c|c|c|c|}
\hline Sr. No. & System & Recall Value & Precision \\
\hline 1. & Modified LexRank & $45.56 \%$ & - \\
\hline 2. & Proposed model & $99.84 \%$ & $98.18 \%$ \\
\hline
\end{tabular}

The proposed model and existing model results (table 4.2) have been measured using the ROUGE system, which has described the proposed model's effectiveness and accuracy to calculate the results of sentiment analysis on the dataset collected from the social media platforms such as Facebook, Twitter, etc. The proposed model has also been evaluated for the precision value which is the probability of the positive predictive value (PPV). The proposed model has been evaluated as the way efficient method than the existing model of sentiment analysis and automatic product review summarization. The new text summarization reflects the real performance of the proposed model.

Table 4.3: The comparison of different compression of sentence compression

\begin{tabular}{|c|c|c|}
\hline \multirow{2}{*}{ Method } & System & Accuracy \\
\hline \multirow{3}{*}{ Pang et. al's } & no_comp_SSC & 88.78 \\
\cline { 2 - 3 } & manual_comp_SSC & 88.04 \\
\cline { 2 - 3 } & auto_comp_SSC & 87.95 \\
\hline \multirow{3}{*}{ Mohammad et. al's } & no_comp_SSC & 91.43 \\
\cline { 2 - 3 } & manual_comp_SSC & 91.06 \\
\cline { 2 - 3 } & auto_comp_SSC & 90.67 \\
\hline \multirow{2}{*}{ Proposed } & no_comp_SSC & 94.14 \\
\cline { 2 - 3 } & manual_comp_SSC & 93.75 \\
\cline { 2 - 3 } & auto_comp_SSC & 96.23 \\
\hline
\end{tabular}


The table 4.2 shows the comparative analysis of the proposed model against the existing models based upon the sentence compression accuracy, which determines the performance of the word list selection or word list filtering in the provided sections. The proposed model has marginally improves the performance by approximately $3-5 \%$ on the basis of each sentence compression domain.

Table 4.4: The comparison between proposed and existing model on various errors

\begin{tabular}{|c|c|c|c|c|}
\hline \multirow[b]{2}{*}{$\begin{array}{l}\text { Paramet } \\
\text { er Type }\end{array}$} & \multicolumn{2}{|c|}{ Existing } & \multicolumn{2}{|c|}{ Proposed } \\
\hline & $\begin{array}{l}\text { Correct } \\
\text { Sentence } \\
\text { Compres } \\
\text { sion } \\
\end{array}$ & $\begin{array}{l}\text { Incorrect } \\
\text { Sentence } \\
\text { Compres } \\
\text { sion } \\
\end{array}$ & $\begin{array}{l}\text { Correct } \\
\text { Sentence } \\
\text { Compres } \\
\text { sion } \\
\end{array}$ & $\begin{array}{l}\text { Incorrect } \\
\text { Sentence } \\
\text { Compres } \\
\text { sion } \\
\end{array}$ \\
\hline $\begin{array}{c}\text { No. of } \\
\text { A-P } \\
\text { Collectio } \\
\text { ns }\end{array}$ & 390 & 118 & 425 & 93 \\
\hline $\begin{array}{c}\text { Error } \\
\text { type 1: } \\
\text { Algorith } \\
\text { m error }\end{array}$ & 15.64 & 17.8 & 10.21 & 14.3 \\
\hline $\begin{array}{c}\text { Error } \\
\text { type 2: } \\
\text { Syntactic } \\
\text { parsing } \\
\text { error }\end{array}$ & 12.31 & 27.12 & 8.86 & 25.02 \\
\hline $\begin{array}{c}\text { Error } \\
\text { type } 3: \\
\text { Compres } \\
\text { sion } \\
\text { error } \\
\end{array}$ & 0 & 20.34 & 0 & 17.54 \\
\hline $\begin{array}{l}\text { Analysis } \\
\text { of } \\
\text { Correct } \\
\text { Results }\end{array}$ & 72.05 & 34.75 & 81.95 & 28.62 \\
\hline
\end{tabular}

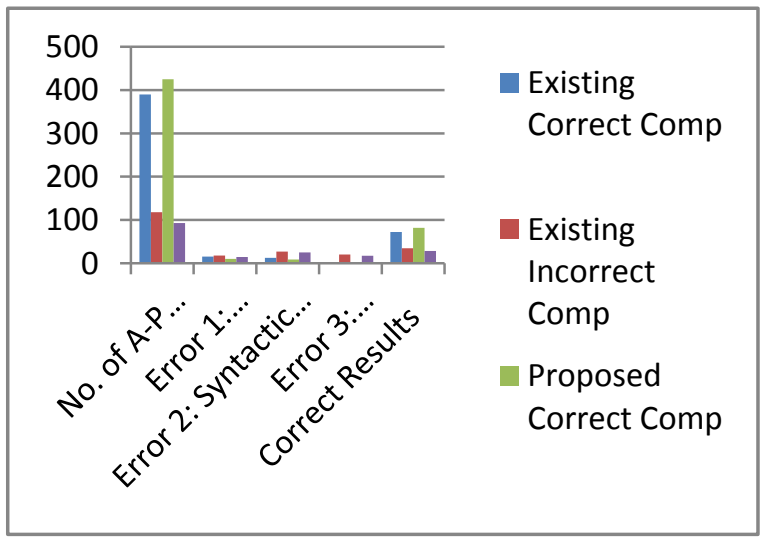

Figure 4.1: The analysis of the proposed model against the existing model parameters

The sentiment analysis errors have been also studied for the evaluation of the proposed model as per shown in the table 4.4. The proposed model has been based upon the new sentiment compression model, which is further evaluated deeply and analyzed for the performance against the existing models. The proposed model has recorded with null value for the compression error, whereas shows the marginal improvements in the algorithmic error and syntactic parsing errors.
The section 5 describes the conclusion obtained from the complete study of sentiment analysis of product reviews and implementing the proposed model.

\section{CONCLUSION}

The proposed model has been designed for the product review classification and polarity evaluation practices. The proposed model is based upon the supervised polarity evaluation and supervised categorization of the message data obtained from the social network for the input product. The supervised categorization and polarity evaluation model utilizes the various keywords lists, which includes the polarity word lists, STOPWORD lists, etc. The proposed model has been evaluated for the results of sentiment analysis using the various performance parameters. The proposed model has been found efficient in the terms of recall, which is recorded nearly at $99.84 \%$. Also the accuracy analysis for correctly processed sentences has been obtained at 72.05 for the existing model against the $81.95 \%$ in the proposed model, which is significantly better than the previous model. The proposed model has consistently outperformed the existing models on the basis of all of the performance measures.In the future, the proposed model can be further extended with the addition of the locally hosted APIs for the live sentiment analysis. Also the new age incorporation of the sentiment analysis may utilize the swarm intelligent algorithms for the analysis of the emoticon weights in the given text data.

\section{REFERENCES}

[1] Che, Wanxiang, Yanyan Zhao, HongleiGuo, Zhong Su, and Ting Liu. "Sentence Compression for Aspect-Based Sentiment Analysis." Audio, Speech, and Language Processing, IEEE/ACM Transactions on 23, no. 12 (2015): 2111-2124

[2] Liu, Siyuan, Xiaoyin Cheng, and Fan Li. "TASC: TopicAdaptive Sentiment Classification on Dynamic Tweets." (2015).

[3] Esuli, Andrea, and FabrizioSebastiani. "Sentiwordnet: A publicly available lexical resource for opinion mining." In Proceedings of LREC, vol. 6, pp. 417-422. 2006.

[4] Kim, Soo-Min, and Eduard Hovy. "Automatic identification of pro and con reasons in online reviews."In Proceedings of the COLING/ACL on Main conference poster sessions, pp. 483-490.Association for Computational Linguistics, 2006.

[5] $\mathrm{Hu}$, Minqing, and Bing Liu. "Mining and summarizing customer reviews."InProceedings of the tenth ACM SIGKDD international conference on Knowledge discovery and data mining, pp. 168-177.ACM, 2004.

[6] Jin, Jian, Ping Ji, and Ying Liu. "Translating online customer opinions into engineering characteristics in QFD: A probabilistic language analysis approach." Engineering Applications of Artificial Intelligence 41 (2015): 115-127

[7] Suanmali, Ladda, NaomieSalim, and Mohammed Salem Binwahlan. "Fuzzy logic based method for improving text summarization." arXiv preprint arXiv:0906.4690 (2009).

[8] Ghorashi, Seyed Hamid, Roliana Ibrahim, ShirinNoekhah, and NiloufarSalehiDastjerdi. "A frequent pattern mining algorithm for feature extraction of customer reviews." In IJCSI International Journal of Computer Science Issues. 2012. 
[9] Yazhini, R., and Raja P. Vishnu. "Automatic summarizer for mobile devices using sentence ranking measure."In Recent Trends in Information Technology (ICRTIT), 2014 International Conference on, pp. 16.IEEE, 2014.

[10] Zha, Zheng-Jun, Jianxing Yu, Jinhui Tang, Meng Wang, and Tat-Seng Chua. "Product aspect ranking and its applications." Knowledge and Data Engineering, IEEE Transactions on 26, no. 5 (2014): 1211-1224.
[11] Jin, Jian, Ping Ji, and Ying Liu. "Prioritising engineering characteristics based on customer online reviews for quality function deployment." Journal of Engineering Design 25, no. 7-9 (2014): 303-324.

[12] Kherwa, Pooja, AnishSachdeva, DhruvMahajan, NishthaPande, and Praveen Kumar Singh. "An approach towards comprehensive sentimental data analysis and opinion mining."In Advance Computing Conference (IACC), 2014 IEEE International, pp. 606-612.IEEE, 2014. 